\title{
Late dislodgement syndrome, which was considered to be the Ratchet syndrome by the surgical removal: complex forces of three syndromes?
}

Haruyuki Kinoshita*, Hiroshi Sugino, Katsunao Tanaka, Takahiro Harada, Kanako Yuasa, Takashi Shimonaga, Orie Ichikawa, Toshiharu Oka and Morihiro Matsuda

Department of Cardiology, Kure Medical Center, 3-1 Aoyama-cho, Kure-shi, Hiroshima 737-0023, Japan

\begin{abstract}
As we reviewed the leads shape transition on chest X-ray, a "twiddler syndrome-type of lead image" was noted, and a couple of months after, a "reel syndrome-type of lead image" was observed. Therefore, we would argue that some cases where dislodgement is observed cannot accurately be categorized as either these syndromes.
\end{abstract}

\section{Introduction}

Ratchet syndrome [1] is caused by a rare malfunction of an implantable device.

In this case, abnormal lead images, such as the images observed in twiddler syndrome [2], and reel syndrome [3], were first noted on imaging over the left breast 1 month and 13 months after pacemaker implantation, respectively. We believe that the dislodgement was due not only to the traction force caused by either rotation of the longer axis or rotation around the shorter axis of the generator but also to the complex force, including a ratchet-like mechanism.

\section{Case report}

An 83-year-old female presented with a chief complaint of dizziness. She had a history of geriatric depression and delirium. A dual-chamber (DDD) pacemaker was implanted into her left breast for sick sinus syndrome (Figure 1A). After 6 months, a change in the position of the atrial lead was observed on chest X-ray (Figures 1B and $1 C$ ). Interrogation of the pacemaker revealed atrial sensing and pacing failure. The pacemaker's generator could be manually rotated easily by $180^{\circ}$ toward the longer axis within a subcutaneous pocket. The patient revealed that she had been repeatedly rotating the generator herself because she was nervous about the area where the pacemaker was implanted. Fortunately, the ventricular lead did not experience sensing and pacing failure. Therefore, we continued to monitor the device over time after changing the pacemaker settings to VVI-mode. After 13 months, dislodgement of the ventricular lead could also be observed. Therefore, we implanted another pacemaker into the right breast. At that time, almost all of the atrial lead was stored in the pocket (in a reel-like manner) (Figure 1D). Next, at the patient's insistence, the DDD pacemaker in the left breast was removed. The fixation between the generator and the greater pectoral muscle was not removed and no loosening was noted. The subcutaneous pocket was not large, but the subcutaneous tissue was sparse, and thus, the surrounding tissue did not cover the generator, providing great mobility within the pocket. ${ }^{4}$ Therefore, the generator could have rotated easily around the fixed portion (Video 1).

\section{Discussion}

In most cases, the type of dislodgement is categorized only by the lead form at the time of removal because a pacemaker is removed when dislodgement of the lead or pacemaker malfunction is observed. In this case, even after atrial lead dislodgement, because the ventricular
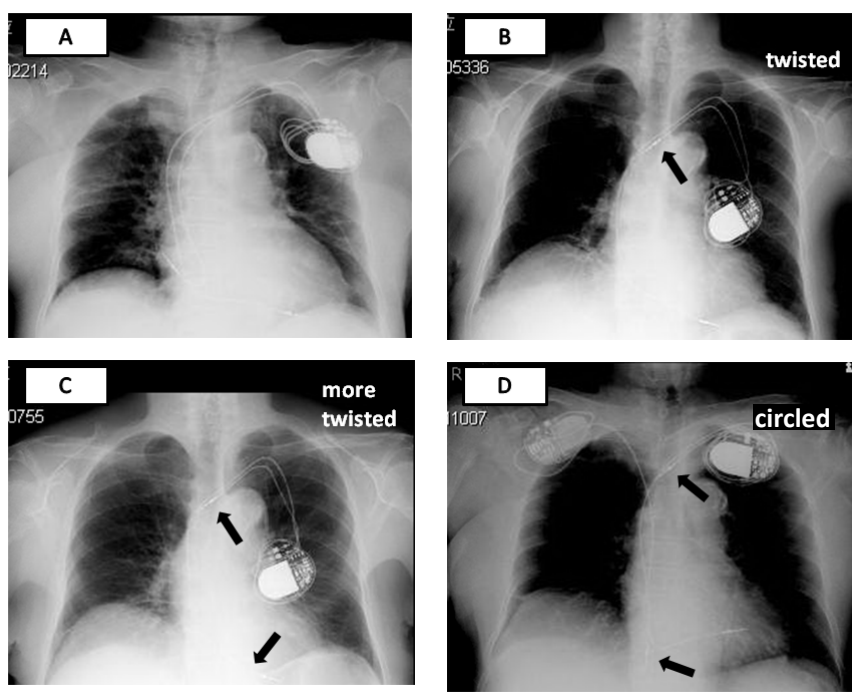

Figure 1. (Video 1) We greatly manipulated the generator from the outside prior to the pacemaker removal surgery. Despite this handling of the pacemaker, the leads did not move a significant amount, even though they did drop out from the cardiac muscle (13 months later).

Correspondence to: Haruyuki Kinoshita, Department of Cardiology, National Hospital Organization, Kure Medical Center, 3-1 Aoyama-cho, Kure-shi, Hiroshima 737-0023, Japan, Tel: +81 82322 3111; Fax: +81 82321 0478; E-mail: haru.kinoshita@gmail.com

Received: October 06, 2016; Accepted: October 19, 2016; Published: October 24,2016 


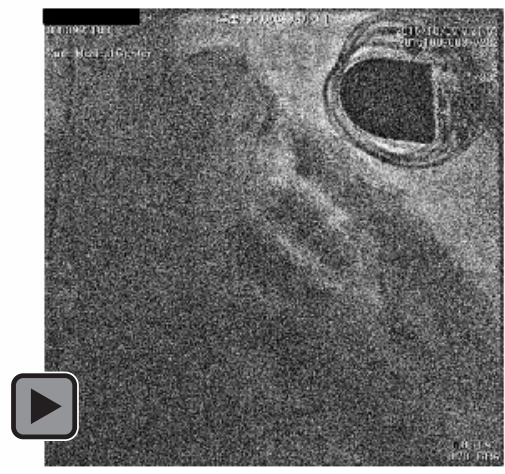

Video 1. We greatly manipulated the generator from the outside prior to the pacemaker removal surgery. Despite this handling of the pacemaker, the leads did not move a significant amount, even though they did drop out from the cardiac muscle (13 months later).

lead was still functioning, we were able to follow the transition of the 'twist' and 'rolling-in' of the lead on chest X-ray over for several months. Like the well-known previously reported cases $[1,2,4]$, such a case could be caused only by rotation around the longitudinal axis or an external force causing rotation around the horizontal axis rotation. However, in this particular case, it was confirmed that fixation between the generator and the greater pectoral muscle was not loosened in the left generator removal surgery. Therefore, it is difficult to determine whether the traction was caused only by the external generator's repeated longitudinal axis rotation or by the horizontal axis, i.e., rotated by the patient herself. In addition, the atrial lead coming out previously ( 6 months later) had no laxity at the fixation point by the sleeve. Meanwhile, the ventricular lead coming out later (13 months after) had a slight laxity of the sleeve [4]. As the lead shape transition on chest X-ray was being reviewed, it must be noted that, after a certain period of traction force around the longitudinal axis, the traction force on the horizontal axis was applied to the lead and that a ratchet-like mechanism could also have been added. Therefore, we concluded that these complex forces would have worked together in a composite manner. Furthermore, the force that was pulling the lead may have been caused by the patient 'actively' exerting force from outside herself [5], or by 'passively' exerting force by the swinging of her breast and changing of her posture or, in some cases, by a combination of those factors. Therefore, as it is difficult to accurately categorize all cases into three types of syndrome, I would like to propose that the syndrome be generically called 'Late dislodgement syndrome'.

\section{Acknowledgments}

The authors would like to thank Wiley's at http:// wileyeditingservices.com for the English language review.

\section{References}

1. Bracke F, van Gelder B, Dijkman B, Meijer A (2005) Lead system causing twiddler's syndrome in patients with an implantable cardioverter-defibrillator. $J$ Thorac Cardiovasc Surg 129: 231-232. [Crossref]

2. 2. Bayliss CE, Beanlands DS, Baird RJ (1968) The pacemaker-twiddler's syndrome: a new complication of implantable transvenous pacemakers. Can Med Assoc J 99: 371373. [Crossref]

3. 3. Carnero-Varo A, Pérez-Paredes M, Ruiz-Ros JA, Giménez-Cervantes D, MartínezCorbalán FR, et al. (1999) "Reel Syndrome": a new form of Twiddler's syndrome? Circulation 100: e45-6. [Crossref]

4. 4. Castillo R, Cavusoglu E (2006) Twiddler's syndrome: an interesting cause of pacemaker failure. Cardiology 105: 119-121. [Crossref]

5. 5. Roberts JS, Wenger NK (1989) Pacemaker twiddler's syndrome. Am J Cardiol 63: 1013-1016.

Copyright: $\mathbb{C} 2016$ Kinoshita H. This is an open-access article distributed under the terms of the Creative Commons Attribution License, which permits unrestricted use, distribution, and reproduction in any medium, provided the original author and source are credited. 